\title{
Importancia de la Flora medicinal promisoria del distrito de Jesús, Cajamarca, Perú
}

\author{
Importance of the Flora promising medicinal of Jesus, \\ Cajamarca, Peru \\ José Mostacero-León ${ }^{2}$, (D) Luis Gilberto García Izquierdo ${ }^{1}$, (D) Anthony De La Cruz Castillo², \\ Manuel Charcape Ravelo , (ID Luis Taramona-Ruíz ${ }^{4}$, (D) Roger Alva Calderón ${ }^{2}$
}

1 Universidad Nacional de Cajamarca. Cajamaca, Perú
${ }^{2}$ Universidad Nacional de Trujillo. Trujillo, Perú
${ }^{3}$ Universidad Nacional de Piura. Piura, Perú
${ }^{4}$ Universidad Le Cordon Bleu. Lima, Perú

Recibido: 10/04/2020 Revisado: 20/05/2020 Aceptado: 21/09/2020 Publicado: 16/12/2020

\section{RESUMEN}

Es indiscutible, el rol que juega la flora y particularmente las plantas medicinales en la mejora de la calidad de vida de la población mundial. En Perú el saber sobre las plantas y sus aplicaciones se ha trasmitido a través de la historia y hoy en día constituye un legado heredado por las comunidades Andino-amazónicas, las cuales son poseedores de una flora excepcional, única y promisoria; y lamentablemente estas tradiciones se están perdiendo paulatinamente, sobre todo por la expansión demográfica. Por lo descrito, esta investigación tiene como objetivo determinar la importancia de la Flora medicinal promisoria de Jesús, Cajamarca, Perú. Para ello, se aplicaron 96 entrevistas semiestructuradas, mediante la técnica "bola de nieve", a fin de recabar datos referentes a caracteres taxonómicos: familia, nombre científico y común, así como caracteres etnomedicinales: parte utilizada, tipo de preparación, forma de administración, enfermedad o dolencia tratada, e índice de Valor de Uso, de cada especie. Se reporta para el distrito de Jesus, 24 especies de flora medicinal promisorias, distribuidas en 23 géneros y 18 familias; siendo, las más representativas por su número de especies, las Asteraceae (3), Lamiaceae (3) y Fabaceas (2). Finalmente, de este total, 18 especies promisorias, fueron consideradas como importantes por el poblador del distrito de Jesus, según su Índice de Valor de uso (IVU). Palabras clave: Caracteres taxonómicos, caracteres etnomedicinales y colectas.

\begin{abstract}
It is indisputable, the role that flora and particularly medicinal plants play in improving the quality of life of the world population. In Peru, knowledge about plants and their applications has been transmitted throughout history and today constitutes a legacy inherited by the Andean-Amazon communities, which are possessors of an exceptional, unique and promising flora; and unfortunately these traditions are gradually being lost, especially due to demographic expansion. Based


on what has been described, this research aims to determine the importance of the promising medicinal flora of Jesus, Cajamarca, Peru. For this, 96 semi-structured interviews were applied, using the "snowball" technique, in order to collect data regarding taxonomic characters: family, scientific and common name, as well as ethnomedical characters: part used, type of preparation, method of administration, treated disease or illness, and Use Value index, of each species. It is reported for the Jesus district, 24 species of Flora promising medical, distributed in 23 genera and 18 families; being, the most representative for their number of species, the Asteraceae (3), Lamiaceae (3) and Fabaceas (2). Finally, of this total, 18 promising species were considered important by the inhabitants of the Jesús district, according to their Use Value Index (IVU). Keywords: Taxonomic characters, ethnomedicinal characters and collections.

\section{INTRODUCCIÓN}

La Flora ha jugado, juega y jugará un Rol preponderante en la vida del ser humano y particularmente, en la del poblador peruano. Este importante recurso no solo sirve como alimento, sino que se utiliza para la obtención de madera, tela, tinte, forraje y medicina; al ser capaz de combatir un sin número de enfermedades y/o dolencias aquejadas por la población desde los albores de la humanidad (Pascual et al., 2014; Castillo et al., 2017; Tello et al., 2019).

El conocimiento ancestral sobre las propiedades de las plantas representa un legado de saber empírico, de acierto y error en el uso correcto y eficaz para la cura de muchas enfermedades padecidas por los pobladores. Los "Hampicamayoc", "Oquetlupuc" y "Sircac" son los poseedores natos de este saber, y se catalogan como verdaderos médicos del Incanato (Dieter y Ortega, 2011; Mostacero et al., 2011).

El conocimiento sobre las propiedades de las plantas trasmitido mediante la palabra limitó su trascendencia en muchas zonas del País y perduró en las Comunidades Andino- Amazónicas específicamente en la memoria de "abuelitas", "brujos", "chamanes" y "emolienteros"; herederos actuales de este saber milenario (Mostacero et al., 2011; Bussmann y Sharon, 2016; Garzón, 2016).
En las comunidades hay especies de flora características de la zona y en muchos casos endémicos desconocidas por muchos distritos, ciudades e incluso departamentos del País. Estas son ricas en vitaminas, sales minerales, carotenoides y pigmentos antociánicos, que sumados a la ingente cantidad de principios activos, como los alcaloides, polifenoles y terpenoides; los constituye, en especies promisorias; pudiendo superar en mucho a las ya comerciales, y así rescatar la importancia de las mismas, en beneficio de las comunidades rurales en particular y del Perú y el mundo en General, toda vez que la creciente expansión demográfica y la consiguiente degradación de sus hábitats, vienen extinguiendo a estas especies promisorias (Horák et al., 2015; Tello et al., 2019).

Muchas, son las investigaciones botánicas que reportan la flora Peruana (Dourojeanni, 1982; Picard y Villar, 1982; Pretell et al., 1985; Sagástegui, 1995) y más específicamente de los Andes y Cajamarca (Ceroni, 2002; Gamarra, 2012; Castañeda y Albán, 2016; García, 2017); por lo que es imprescindible que Biólogos, Etnobotánicos y Químico Farmaceúticos, continúen explorando e investigando, en estas comunidades, como fuentes de este saber milenario; a fin de rescatarlo, revalorar y redescubrir los principales atributos medicinales que poseen 
estos recursos (Mostacero et al., 2009; Mostacero et al., 2017a; Bussmann y Sharon, 2015). Por lo antes descrito y teniendo en cuenta que no hay precedentes de investigaciones de índole etnomedicinal en el distrito de Jesús. Esta investigación se avocó a determinar la determinar la importancia de la Flora medicinal promisoria de Jesús, Cajamarca, Perú.

\section{MATERIAL Y MÉTODOS}

\section{Área de estudio}

La presente investigación se desarrolló en el distrito de Jesús, provincia Cajamarca, Perú. Ubicado a $07^{\circ} 14^{\prime} 44^{\prime \prime}$ Latitud Sur; y $78^{\circ} 22^{\prime} 40^{\prime \prime}$ longitud Oeste; a una altitud de 2531 m.s.n.m

\section{Muestra}

La muestra estuvo constituida por 96 personas, dato obtenido, mediante la fórmula propuesta por Bocanegra et al. (2011), que considera población finita $\mathrm{y}$ varianza desconocida, con un error permisible de $10 \%$ y un nivel de confianza del $95 \%$.

$$
\mathbf{n}=\frac{\mathrm{N} Z^{2} p q}{d^{2}(N-1)+Z^{2} p q}
$$

Donde:

$\mathrm{n}$ : tamaño de la muestra; N: población del estudio ( $\mathrm{n}=14792$ habitantes); p: proporción de éxito de la característica de interés, igual a $50 \%(0.5)$; $\mathrm{q}: 1-\mathrm{P}=$ complemento de P; $\alpha / 2$ Z: coeficiente de confiabilidad al $95 \%$ igual a 1.96; d: tolerancia de error permisible en la investigación $=(10 \%)$.

$$
n=\frac{(14792)\left(1.96^{2}\right)(0.5)(0.5)}{0.1^{2}(14792-1)+1.96^{2}(0.5)(0.5)}=96
$$

\section{Instrumento y Técnicas de recolección de datos}

Se empleó como instrumento de recolección de datos a la entrevista semiestructurada. Para ello se utilizó la técnica "bola de nieve" (Bailey, 1994); en donde, se procedió a entrevistar al primer individuo conocedor de las plantas medicinales, en el distrito de Jesús, quien brindó información requerida acerca de los nombres vulgares, parte empleada de la planta, forma de administración, así como los usos medicinales que les confieren a cada espécimen. Al finalizar la entrevista, se le solicitó que indicara el nombre otra persona a entrevistar conocedora del tema. Luego se realizó la entrevista a la segunda persona que al igual que la anterior, proporcionó información concerniente a plantas medicinales; y así, a modo de "bola de nieve" se aplicaron los 94 entrevistados restantes, a fin de respaldar y contrastar la información antes obtenida.

\section{Colecta e Identificación de las plantas}

La colecta de las plantas se realizó paralelamente a la aplicación de las entrevistas, y en compañía constante de una de las personas conocedoras de la zona. Los materiales recolectados se transportaron en prensa botánica para su herborización e introducción al Herbarium Truxillense de la U.N.T (H.U.T.); donde se procedió a secarlos, montarlos, etiquetarlos, preservarlos y registrarlos. También, se hizo uso de claves taxonómicas referidas a la flora peruana, según: Brako y Zarucchi (1993) y Mostacero et al. (2009), agenciados con los portales virtuales: Trópicos y The Plant List.

\section{Análisis de datos}

La información obtenida, fue analizada, organizada, complementada y contrastada, con la información hallada en tesis, artí- 
culos científicos y libros sobre la materia. De igual manera a fin de expresar la importancia o valor cultural atribuida a estas especies promisorias, se determinó el índice de valor de uso de cada especie (IVU), la que fue estimada según la fórmula propuesta por Zambrano et al. (2015):

Dónde:

$$
I V U=\frac{V U_{i s}}{N_{s}}
$$

$\mathrm{VU}_{\text {is }}$ : Valor de uso de la especie por cada informante; $\mathrm{N}_{\mathrm{s}}$ : Número de informantes para cada especie. Cabe destacar que el valor de este índice oscila entre 0 y 1 ; tomando como especie importante aquella cuyo IVU sea mayor a 0.5. Finalmente, a través del trabajo de gabinete, se contrastó analíticamente toda la información obtenida, se organizó y se redactó el informe final.

\section{RESULTADOS Y DISCUSIÓN}

En la Tabla 1, se detallan datos referentes a los caracteres taxonómicos: familia, nombre científico y común, así como los caracteres etnomedicinales: parte utilizada, tipo de preparación, forma de administración, enfermedad o dolencia tratada, e índice de Valor de Uso, de la flora medicinal, empleada por la población del distrito de Jesús, Cajamarca. Consistente en 24 especies; las mismas que se encuentran distribuidas en 18 familias y 23 géneros (figura 1). Sumado a ello, en la (figura 2), se muestran las familias más representativas empleadas por el poblador de este distrito.

Tabla 1. Caracteres taxonómicos y etnomedicinales de la flora utilizada por el poblador del distrito de Jesús, Cajamarca, Perú

\begin{tabular}{|c|c|c|c|c|c|c|c|c|}
\hline $\mathrm{N}$ & NOMBRE CIENTÍFICO & $\begin{array}{l}\text { NOMBRE } \\
\text { COMÚN }\end{array}$ & $\begin{array}{c}\text { PARTE } \\
\text { UTILIZADA }\end{array}$ & $\begin{array}{c}\text { TIPO DE } \\
\text { PREPARADO }\end{array}$ & $\begin{array}{l}\text { MODO DE } \\
\text { APLICACIÓN }\end{array}$ & USO & RU & IVU \\
\hline 1 & $\begin{array}{l}\text { Adiantum subvolubile Mett. } \\
\text { ex Kuhn PTERIDACEAE }\end{array}$ & "culantrillo" & rizomas & Cocimiento & Oral & $\begin{array}{l}\text { Acción colagoga, } \\
\text { depurativa y sedante }\end{array}$ & 56 & 0.58 \\
\hline 2 & $\begin{array}{l}\text { Alternanthera vellosa Kunth } \\
\text { AMARANTHACEAE }\end{array}$ & "flor de arena" & $\begin{array}{l}\text { tallo y } \\
\text { hojas }\end{array}$ & Cocimiento & Oral & $\begin{array}{l}\text { Desinflamante de las } \\
\text { vías urinarias, elimina } \\
\text { cálculos y arenillas } \\
\text { renales. }\end{array}$ & 68 & 0.71 \\
\hline 3 & $\begin{array}{l}\text { Ambrosia peruviana Willd. } \\
\text { ASTERACEAE }\end{array}$ & $\begin{array}{l}\text { "marco", } \\
\text { "altamisa" }\end{array}$ & $\begin{array}{l}\text { tallo y } \\
\text { hojas }\end{array}$ & Cocimiento & Oral & $\begin{array}{l}\text { Acción antirreumática, } \\
\text { antiespasmódica y } \\
\text { contra la dismenorrea. }\end{array}$ & 70 & 0.73 \\
\hline 4 & $\begin{array}{l}\text { Arcytophyllum thymifolium } \\
\text { (Ruiz \& Pav.) Standl. } \\
\text { RUBIACEAE }\end{array}$ & "escobilla" & $\begin{array}{l}\text { tallo y } \\
\text { hojas }\end{array}$ & Cocimiento & Oral & $\begin{array}{c}\text { Desinflamante del tracto } \\
\text { gastrointestinal }\end{array}$ & 41 & 0.43 \\
\hline 5 & $\begin{array}{l}\text { Bixa orellana } \mathrm{L} \text {. } \\
\text { BIXACEAE }\end{array}$ & "achiote" & hoja & $\begin{array}{l}\text { Infusión y } \\
\text { cocimiento }\end{array}$ & Oral & $\begin{array}{l}\text { Acción antiinflamatoria, } \\
\text { contra la prostatitis }\end{array}$ & 71 & 0.74 \\
\hline 6 & $\begin{array}{l}\text { Buddleja americana L. } \\
\text { SCROPHULARIACEAE }\end{array}$ & "flor blanca" & $\begin{array}{l}\text { tallo y } \\
\text { hojas }\end{array}$ & $\begin{array}{l}\text { Infusión y } \\
\text { cocimiento }\end{array}$ & Oral & $\begin{array}{l}\text { Acción colagoga y } \\
\text { sedante }\end{array}$ & 53 & 0.55 \\
\hline
\end{tabular}




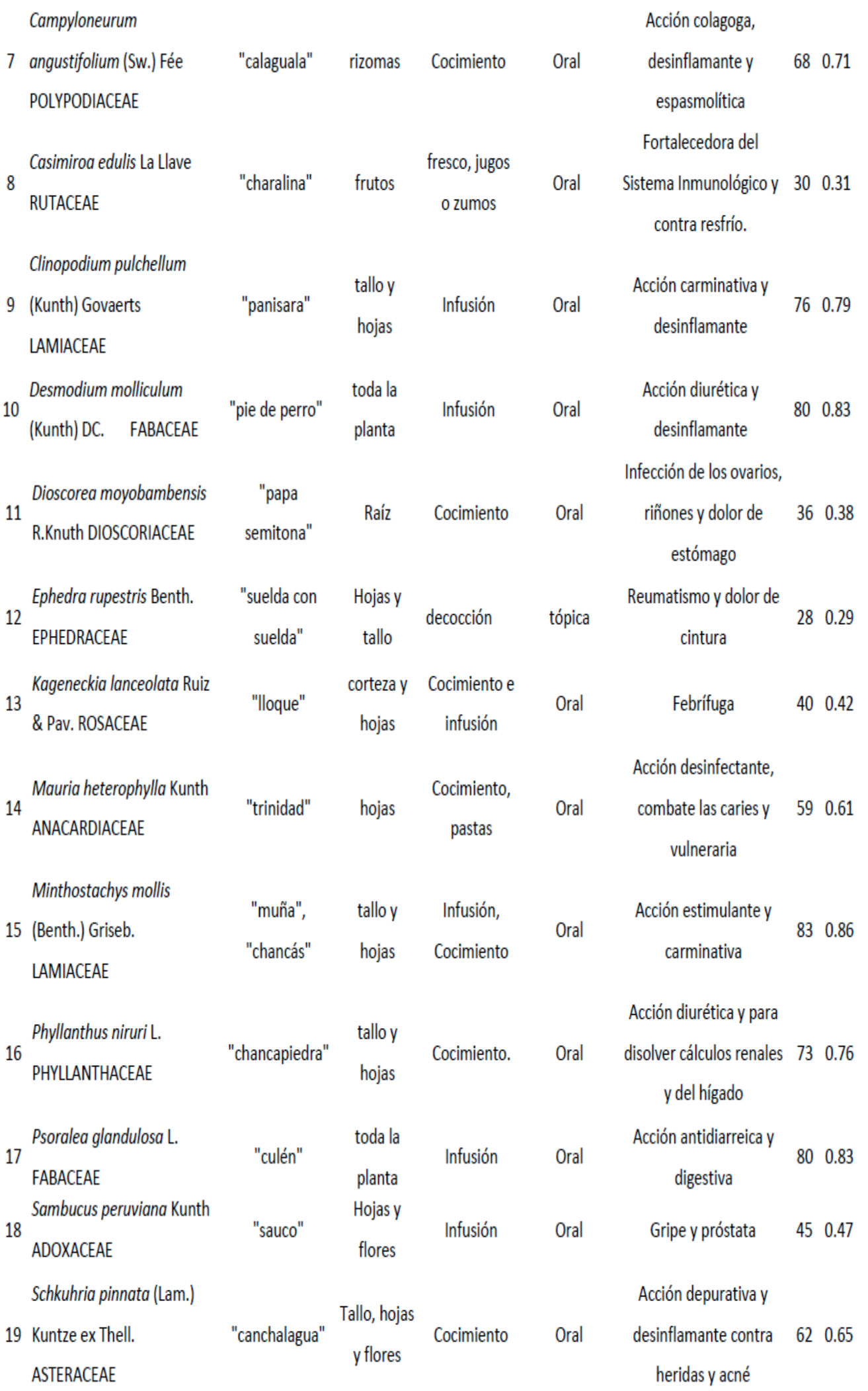




$20 \begin{aligned} & \text { Siparuna subinodora (Ruiz \& } \\ & \text { Pav.) A. DC. SIPARUNACEAE }\end{aligned}$
Smañasquero"
21 (Poepp.) H.Rob.
ASTERACEAE

Stachys arvensis (L.) L. "supiquehua"
LAMIACEAE

23

Valeriana officinalis L. "valeriana"
CAPRIFOLIACEAE
Tallo y
hojas

raices

tuberosas

planta

raíces

tuberosas

raíces

tuberosas

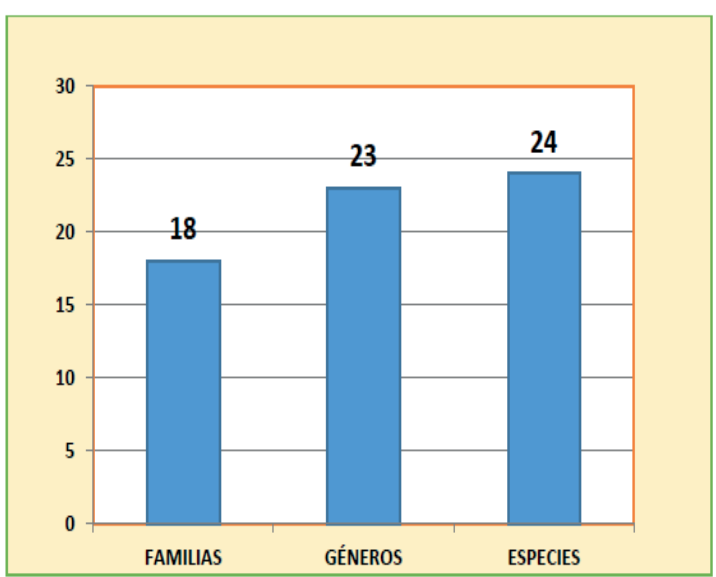

Figura 1. Distribución en familias, géneros y especies, de la flora utilizada por el poblador del distrito de Jesús, Cajamarca, Perú

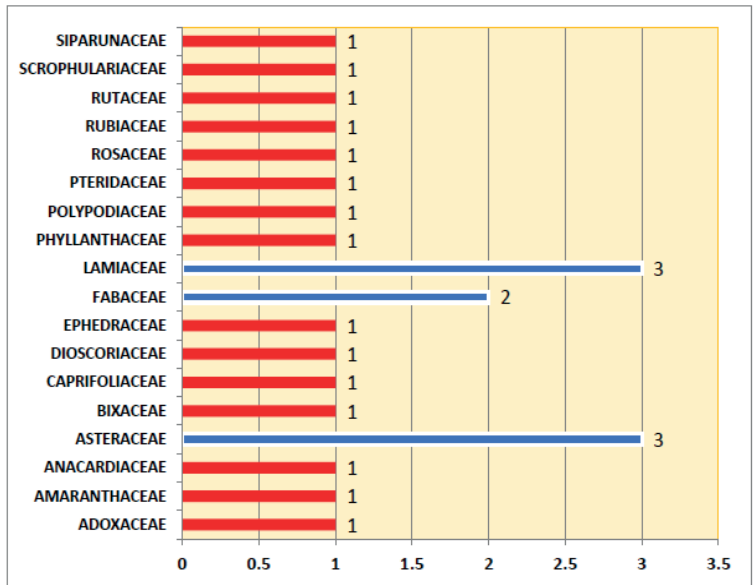

Figura 2. Familias más representativas de flora empleadas por el poblador del distrito de de Jesús, Cajamarca, Perú

\begin{tabular}{ccc} 
Extractos & \multicolumn{3}{c}{ Mal de espanto, } \\
acuososy & Tópico & febrífugoy \\
etanólicos & & 6306 \\
enfermedades de la piel &
\end{tabular}

Oral Contra la diabetes $90 \quad 0.94$

Infusión Oral Acción carminativa 490.51

Combate el estrés, el

Infusión,

cocimiento

Oral

dolor de cabeza, la

esquizofrenia ye

730.76

insomnio

Infusión,

cocimiento

Oral

Nervios y el insomnio $\quad 70 \quad 0.73$

En la tabla 1, se muestra un total de 24 familias de flora medicinal promisorias, reportadas en el distrito de Jesús, y por consiguiente empleada por estos pobladores; cabe resaltar que dichas especies se encuentran distribuidas en 23 géneros y 18 familias (Figura 1). Las familias más representativas son Asteraceae, Lamiaceae y Fabaceae poseen 3, 3 y 2 especies respectivamente (Figura 2). Tales datos, concuerdan con lo investigado por $\mathrm{La}$ Torre y Albán (2006), Gonzales et al. (2014), Angulo et al. (2012); quienes atribuyen que dicha representatividad, especialmente de las familias Asteraceae y Lamiaceae, se debe al hecho de ser cosmopolitas, lo que facilita su disponibilidad; sumado a la ingente cantidad de metabolitos secundarios; vitaminas, sales minerales y antioxidantes, depositados ya sea en sus raíces, tallos, hojas o flores; y que les confiere propiedades terapéuticas; capaces de curar y/o aliviar múltiples enfermedades; lo que las conlleva a ser las más expendidas en mercados de diversas ciudades del país (Huamantupa et al., 2011; Osorio, 2009; Castillo et al., 2017; Mostacero et al., (2019). 
Por otro lado, del total de especies promisorias para la comunidad de Jesús, Cajamarca; las más importantes por su IVU, son: $S$. sonchifolius (IVU=0.94), M. mollis (IVU=0.86), D. molliculum (IVU=0.83), $P$. glandulosa (IVU=0.83), C. pulchellum (IVU= 0.79), $P$. niruri (IVU=0.76), $V$. officinalis (IVU= $0.76), B$. orellana $(\mathrm{IVU}=0.74), A$. peruviana (IVU=0.73)", $V$. pinnatifida (IVU=0.73), $A$. villosa (IVU=0.71), C. angustifolium (IVU= $0.71), S$. subinodora (IVU=0.66), $S$. pinnata $(\mathrm{IVU}=0.65), M$. heterophylla $h(\mathrm{IVU}=0.61)$, $A$. subvolubile $(\mathrm{IVU}=0.58), B$. americana $(\mathrm{IVU}=0.55)$ y $S$. arvensis $(\mathrm{IVU}=0.51)$. Esta flora que ha sido empleada por la población local del distrito; en muchos casos desconocidas a nivel nacional e internacional; pero que ha logrado suplir a lo largo de la historia, un sin número de enfermedades; como las relacionadas a los sistemas: digestivo, nervioso, respiratorio, cardiovascular, genitourinario, músculo esquelético, además de tratar innumerables afecciones atribuidas a maleficios (Tabla 1). Es tarea de biólogos, antropólogos, químicos farmaceúticos y sociólogos revalorar los usos y costumbres que tienen esta comunidad rural, a fin de rescatar ese valioso legado ancestral y sentar las bases para futuras investigaciones del tipo aplicadas. Estas especies se han adaptado a las inclemencias del ambiente a la par de adquirir muchos metabolitos secundarios y principios activos, que de ser investigados y validados científicamente, podrían resultar en la formulación de nuevos fármacos, que superarían en mucho a los ya comerciales; generando sustentabilidad económica para el poblador local del Perú; hecho que concuerda en lo reportado por Magaña et al. (2010), Mostacero et al. (2011), Horák et al. (2015).

A la par de lo descrito, en los párrafos anteriores, y concordando con lo descrito por Mostacero et al. (2011) y Acosta (2012) urge también empezar a incentivar proyectos, vinculados a restaurar el hábitat natural de estas especies medicinales promisorias. Los que día a día se ven afectados además de la expansión demográfica; así como por su colecta desmesurada; por ello es misión de las instituciones gubernamentales, así como de las instituciones de educación superior, fomentar la conservación de estos recursos, toda vez que la salud es una de las necesidades primordiales para el hombre, y particularmente en Jesús, la población, no cuenta con los recursos económicos como para utilizar un servicio médico particular.

\section{CONCLUSIONES}

Se reporta para el distrito de Jesús, 24 especies de flora medicinal promisorias, distribuidas en 23 géneros y 18 familias; siendo las Asteraceae (3), Lamiaceae (3) y Fabaceas, las más representativas, por su número de especies.

Del total de especies reportadas, 18 resultan imprescindibles; según su índice de Valor de Uso, para el poblador de Jesús, en la cura y/o tratamiento de sus enfermedades; por lo que urge rescatar este saber ancestral, a la par de servir de base para futuras investigaciones del tipo aplicadas, buscando en todo momento la sustentabilidad de estos recursos a fin de mejorar la calidad de vida del poblador de Jesús en particular y del Perú y del mundo en general.

\section{REFERENCIAS BIBLIOGRÁFICAS}

Angulo, A.; Rosero, R.; González, M. (2012). Estudio etnobotánico de las plantas medicinales utilizadas por los habitantes del corregimiento de Genoy, Municipio de Pasto, Colombia. Rev. Universidad y Salud. 14(2):168-185

Acosta, L. (2012). Plantas medicinales en un proyecto de desarrollo humano. Rev Cubana Plant Med, 17 (4): 446-451. 
Bailey, K. (1994). Methods of Social Research. Ed. The Free Press, New York, USA.

Bocanegra, L.; Bocanegra, F.; Mostacero, J. (2011). Efectividad de la medicina herbolaria y su impacto en la calidad de vida de los pobladores de Curgos, Perú. UCV - Scientia 3(1):23-34.

Brako, L.; Zarucchi J. (1993). Catálogo de las Angiospermas y Gimnospermas del Perú. Monogr. Syst. Bot. Missouri Bot. Garden. Vol 45.

Bussmann, R.; Sharon, D. (2015). Plantas medicinales de los Andes y la Amazonia: la flora mágica y medicinal del norte del Perú. Centro Willian L. Brown-Jardin Botánico de Missouri.

Bussmann, R.; Sharon, D. (2016). Plantas medicinales de los Andes y la Amazonía - La flora mágica y medicinal del Norte del Perú Ethnobotany Research \& Applications 15(1):1-293.

Castañeda, R.; Albán, J. (2016). Importancia Cultural de la flora silvestre del distrito de Pamparomás, Ancash, Perú. Ecología Aplicada. 15 (2): 151-169.

Castillo, A.; Suárez, J.; Mosquera, J. (2017). Naturaleza y Sociedad: Relaciones y tendencias desde un enfoque $\mathrm{Eu}-$ rocéntrico. rev.luna.azúl; 44: 348-371.

Castillo-Vera, H.; Cochachin, E.; Albán, J. (2017). Plantas comercializadas por herbolarios en el mercado del distrito de Cajabamba (Cajamarca, Perú) Bol Latinoam Caribe Plant Med Aromat, 16 (3): 303-318

Ceroni, A. (2002). Datos etnobotánicos del poblado de Huaylingas, Cuenca La Gallega. Morropón. Piura. Ecología Aplicada. 1 (1): 65-70.
Dieter, K.; Ortega, F. (2011). La botica de Dios: plantas medicinales y su uso. Ed. Trama ediciones Imprenta Mariscal, Quito, Ecuador.

Dourejeanni, M. (1982). Recursos Naturales y Desarrollo en América Latina y el Caribe. Edit. Universidad de Lima. Lima, Perú.

Gamarra, P. (2012). "Estudio etnobotánico del distrito de Marca, Recuay - Ancash". Tesis para optar al grado de Magister. UNMSM, EPG. Lima - Perú. 269 pp.

García, F. (2017). Etnobotánica de cuatro comunidades del distrito de Huambos, Cajamarca". Tesis para optar el Título Profesional de Bióloga. Universidad Nacional Agraria La Molina. Lima - Perú.

Garzón, P. (2016). Conocimiento Tradicional sobre las plantas medicinales de yarumo ( $\mathrm{Ce}$ cropia sciadophylla), carambolo (Averrhoa carambola) y uña de gato (Uncaria tomento$s a$ ) en el resguardo indígena de Macedonia, Amazonas. revista.luna.azúl; 43: 386-414.

González, M.; Malpartida, S.; Beltrán, H.; Jullian, V.; Bourdy, G. (2014). Hot and Cold: Medicinal plant uses in Quechua communities in the high Andes (Callejón de Huaylas, Ancash, Perú). Journal of Ethnopharmacology, 155(2):1-25.

Horák, M.; Somerlíková, K.; Kavenská, V.; Granda, L.; Škrabáková, L.; Tournon, J.; Chuspe, M.; Rosero, M.; Forero, L.; Rosero, A.; Castro, N.; Beltrán, G.; Halbich, M.; Minero, F.; Rosas, I.; Mateos, E. (2015). Etnobotánica y fitoterapia en América. Horák M. (Editor). Universidad de Mendel en Brno, Facultad de Desarollo Regional y Estudios 
Internacionales, Departamento de Idiomas y Estudios Culturales. República Checa.

Huamantupa, I.; Cuba, M.; Urrunaga, R.; Paz, E.; Ananya, N.; Callalli, M.; Pallqui, N.; Coasaca, H. (2011). Riqueza, uso y origen de plantas medicinales expendidas en los mercados de la ciudad del Cusco. Rev Peru Biol 18: 283 - 291.

La Torre, M.; Albán, J. (2006). Etnobotánica en los Andes del Perú. En: Morales, M.; Ollgaard, L.; Kvist, L.; Borchsenius, B.; Balslev, H. Botánica Económica de los Andes Centrales. pp. 239-245. Universidad Mayor de San Andrés, La Paz, Bolivia.

Magaña, M.; Gama, L.; Mariaca, R. (2010). El Uso de las Plantas Medicinales en las Comunidades Mayachontales de Nacajuca, Tabasco, México. Polibotánica, 29: 213-262.

Mostacero, J.; Castillo, F.; Mejía, F.; Gamarra, O.; Charcape, J.; Ramírez, R. (2011). Plantas Medicinales del Perú: Taxonomía, Ecogeografía, Fenología y Etnobotánica. Trujillo- Perú: AsambleaNacionaldeRectoresFondoEditorial.

Mostacero, J.; Mejía, F.; Gamarra, O. (2009). Fanerógamas del Perú: Taxonomía, utilidad y ecogeografía. CONCYTEC. Trujillo- Perú.

Mostacero, J.; Mejía，F.; Gastañadui， D.; De La Cruz, J. (2017a). Inventario taxonómico, fitogreográfico y etnobotánico de frutales nativos del norte del Perú. Scientia Agropecuaria 8 (3): 215 - 224.

Mostacero, J.; Peláez, F.; Alarcón, N.; De La Cruz, A.; Alva, R.; Charcape, M. (2019). Plantas utilizadas para el tratamiento del cáncer expendidas en los principales mercados de la provincia de Trujillo, Perú, 2016-2017. Bol Latinoam Caribe PlantMed Aromat, 18(1):81-94.

Osorio, E. (2009). Aspectos básicos de farmacognosia. TESIS. Facultad de Química Farmacéutica. Universidad de Antioquia. Colombia. 83 pp.

Pascual-Casamayor, D.; Pérez- Campos, Y.; Morales- Guerrero, I.; Castellanos- Coloma, I.; González- Heredia, E. (2014). Algunas consideraciones sobre el surgimiento y la evolución de la medicina natural y tradicional. MEDISAN;18(10):1467-74.

Picard, L.; Villard, M. (1982). Metodología Utilizada Para la Introducción de Especies Forestales en Cajamarca - Perú. Edit. Centro de Investigación y Capacitación Forestal. CICAFOR, Cajamarca, Perú.

Pretell, J.; Ocaña, R.; Barahona, E. (1985). Apuntes Sobre Algunas Especies Forestales Nativas de la Sierra Peruana. Proyecto FAO/ Holanda/INFOR. Edit. Centauro S.A. Lima, Perú. Sagástegui, A. 1995. Diversidad Florística de Contumazá. Edit. Libertad, Trujillo, Perú.

Tello-Ceron, G.; Flores- Pimentel, M.; Gómez- Galarza, V. (2019). Uso de las plantas medicinales del distrito de Quero, Jauja, Región Junín, Perú. Ecol. apl. 18(1): 11-20.

Zambrano, L.; Buenaño, M.; Mancera, N.; Jiménez, E. (2015). Estudio etnobotánico de plantas medicinales utilizadas por los habitantes del área rural de la Parroquia San Carlos, Quevedo, Ecuador. Rev.Univ. Salud 17:97 - 111. 